\title{
Sampled-Data Control with Adjustable Switching Frequency for DC-DC converters
}

\author{
Xingda Yan, Member, IEEE, Zhan Shu, Senior Member, IEEE, Suleiman M. Sharkh, Senior Member, IEEE \\ Zheng-Guang Wu Member, IEEE, and Michael Z. Q. Chen, Senior Member, IEEE
}

\begin{abstract}
In this paper, a novel sampled-data control approach is proposed for DC-DC converters. The DC-DC power electronic converter is modeled as a sampled-data switched affine system according to the status of the power switch. A novel switching control algorithm is synthesized by using the switched Lyapunov theory. The proposed approach is able to not only drive the output to a prescribed set point from any initial condition but also track a varying reference signal, and the switching frequency can be adjusted online with guaranteed stability. In addition, with this approach, CCM and DCM operations can be treated in a unified way. The effectiveness and merits of the proposed method are illustrated by experiments on a laboratory prototype.
\end{abstract}

Index Terms-DC-DC conversion, sampled-data control, variable switching frequency

\section{INTRODUCTION}

D C-DC power electronics converters have been widely used in practical applications, e.g., power supplies [1], DC motor drives [2], portable electronic devices [3], avionic systems [4] and RF transmitters [5], [6], etc. One of the most popular control techniques used for DC-DC converters by far is the linear control methods based on the small-signal averaging model proposed by Middlebrook in the 1970s [7] and the Pulse Width Modulation (PWM) technique [8]. Although these conventional control approaches may be adequate in some cases, the controller performance is quite limited, and the design involves a number of heuristic procedures, which lack theoretical guarantees. This motivates the study of advanced control technology for DC-DC converters, and a large number of control methods have been developed or tailored to control problems of DC-DC converters, e.g., sliding mode control [9], [10], fuzzy logic control [11], adaptive control [12], robust control [13] model predictive control [14], [15], artificial neural network control [16], Lyapunov-based control [17], [18], to just mention a few.

Manuscript received Jul 10, 2017; revised Mar 02, 2018, revised Jun 17, 2018; accepted Oct 09, 2018.

This work was partially supported by China Scholarship Council (CSC), Annual Adventure in Research Grant (University of Southampton), and NSFC under 61403173. (Corresponding authors: Zhan Shu).

X. Yan, Z. Shu and S. M. Sharkh are with Mechanical Engineering Department, University of Southampton, Southampton, UK, SO17 1BJ Email:X.Yan@soton.ac.uk.

Z. Wu is with the Institute of Cyber Systems and Control, Zhejiang University, Zhejiang, China.

Michael Z. Q. Chen is with the Department of Mechanical Engineering, The University of Hong Kong, Pokfulam Road, Hong Kong.
Despite the effectiveness of these approaches, there are still a number of challenges that need to be tackled. In some applications, the output voltage of DC-DC power converter are required to track a changing reference signal or adjust manually, e.g., the power system of microprocessors [19] or the power supply of a variable envelope radio frequency power amplifier [20], [21]. Many small-signal control approaches may not perform well for this scenario and even become unstable [22], since the controller is usually designed based on a model linearized or discretized at an operating point of interest. For large-signal control design, quantitative characterizations on stability, robustness, and performance are usually unavailable, and their stability, robustness, and performance need to be evaluated through experiment. In addition, digital implementation of most nonlinear controllers remains a problem.

Another issue in the design of DC-DC converters is the tradeoff between performance and efficiency. For fixed switching frequency control design, fast dynamic response and low output ripples can be achieved by adopting a high switching frequency. However, over-rapid switching will impair energy efficiency and pose a challenge to thermal management [23]. And the switching load does not scale with load [24] which results in poor light load efficiency. Therefore, variable switching frequency based controller has been adopted to tackle this issue. For instance, in battery-operated systems, variable frequency operation is shown to give better energy efficiency and provide wider rage of usable power [25]. Although there are some preliminary results along this direction [26], [27], the problem has not been fully investigated.

In this paper, the DC-DC converters are considered to be controlled by a digital controller. A sampled-data switched model is established to describe the closed-loop dynamics. Our goal is to design a switching controller with guaranteed closed-loop stability in the entire state space for varying reference points. Comparing with the existing results, the main contributions of this paper are summarized as:

1) The proposed sampled-data switched model captures fulltime information instead of a discrete-time model without the inter-sample behaviour of the power electronic converters, and the switching controller can be implemented easily with digital devices.

2) The designed controller is able to track changing reference signals without re-design, and this is guaranteed theoretically. 
3) Switching frequency can be adjusted online without losing closed-loop stability.

4) The parameters of the switching controller is calculated offline, thus significantly reducing the online computation burden.

5) CCM and DCM operations can be treated in a unified approach.

This paper is organized as follows. In Section II, a unified switched system model for a class of DC-DC converters is established. In Section III, a switching control law is designed off-line by solving a set of matrix inequalities. The closedloop stability with the designed controller is also established through the use of the switched Lyapunov theory. In Section IV, a practical DC-DC buck converter is used as an example for case study. A laboratory prototype is employed to show the effectiveness and merits of the proposed approach. In the end, conclusions are drawn in Section V, and the proofs of all the theorems and corollaries are presented in Appendix.

Notation: The transpose of a matrix $P$ is denoted as $P^{T}$. The symbol $*$ denotes a block that can be inferred by symmetry. For a square symmetric matrix, $P>0(P<0)$ means that $P$ is positive definite (negative definite). For a piecewise function $\sigma \triangleq \arg \min \left(f_{i}(x)\right)(i \in\{1,2\}), \sigma=1$ if $f_{1}(x)<f_{2}(x)$ and $\sigma=$ 2 if $f_{2}(x)<f_{1}(x)$. $\operatorname{eig}_{\min }(P)$ is the minimum eigenvalue of matrix $P$. The symbol $\operatorname{co}\{\Psi\}$ denotes the convex hull of the set $\Psi$.

\section{SWITCHED MOdEL FOR DC-DC CONVERTERS}

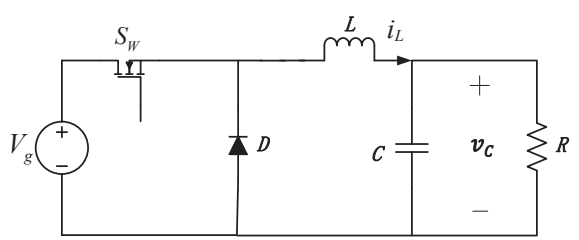

Figure 1: Topology of DC-DC Buck Converters

In this section, a switched model of DC-DC buck converters is presented. The topology of a non-isolated DC-DC buck converter is shown in Fig. $1 . V_{g}$ is the source DC voltage; $L$ is the inductance of a inductor; $C$ is the capacitance of a capacitor; and $R$ represents load resistance. The current through the inductor and the voltage across the capacitor are $i_{L}$ and $v_{C}$ respectively. Due to the space limit, the modelling and control design is focused on the DC-DC buck converters. However, it should be stressed that the results could be extended to different DC-DC topologies with no extra effort as the switched models for different topologies have the same structure.

For each switching state, the continuous dynamics of the converter can be described by a state-space model:

$$
\dot{x}(t)=A_{\sigma} x(t)+B_{\sigma}, \sigma \in \Xi \triangleq\{1,2\}
$$

where

$$
A_{1}=A_{2}=\left[\begin{array}{cc}
0 & -\frac{1}{L} \\
\frac{1}{C} & -\frac{1}{R C}
\end{array}\right] \quad B_{1}=\left[\begin{array}{c}
\frac{V_{g}}{L} \\
0
\end{array}\right] \quad B_{2}=\left[\begin{array}{l}
0 \\
0
\end{array}\right]
$$

with the system states $x(t)=\left[i_{L}, v_{C}\right]^{T} \in \mathbb{R}^{2} .\left(A_{1}, B_{1}\right)$ and $\left(A_{2}, B_{2}\right)$ are the system matrices for the ON state and OFF state respectively.

Due to the operation of the transistor $S_{W}$, the evolution of the system state is determined by the continuous dynamics together with the status of the switch $(\sigma=1$ for $\mathrm{ON}$ or 2 for OFF). It is stressed here that although similar models have been used for the simulation of DC-DC converters, control design, particularly sampled-data control, based on the switched model remains rare.

From the standpoint of control theory, the system in (1) has discontinuous vector field, and thus classic equilibrium solution is no longer applicable. However, our goal is to regulate the output voltage to some reference points, which are some sorts of "equilibria". To solve this issue, we employ the so-called Filippov solutions to obtain the attainable reference points [28]. The basic idea is to replace the right-hand side of (1) with a differential inclusion, that is,

$$
\dot{x}(t)=A(\lambda) x(t)+B(\lambda),
$$

where $A(\lambda)=\lambda A_{1}+(1-\lambda) A_{2}, B(\lambda)=\lambda B_{1}+(1-\lambda) B_{2}$, and $\lambda \in[0,1]$. With this "averaged" system, the attainable equilibrium can be obtained as

$$
x_{e}=\left[i_{L, e}, v_{C, e}\right]^{T}=-A^{-1}(\lambda) B(\lambda), \lambda \in[0,1] .
$$

\section{Sampled-data Controller Design}

A significant difference between the proposed method and the most digital control schemes for DC-DC converters such as DPWM [29] and model predictive control [14] is that: in order to capture more transition information of the converters, the design is based on a continuous-time plant (1) with full-time information rather than a discrete-time model that neglects the inter-sample behaviour of the converters. On the other hand, the proposed method is considerably different from the sliding mode control [9] [10], which is also commonly applied in power converters control and is based on a large signal representation of the system. The attractive properties of the sliding mode control lie in the fact that the design procedure is straightforward and it is robust to parameters variations. However, the main drawback is its high switching frequency. For the proposed method in this paper, the switching frequency is bounded by the sampling frequency and even tunable based on the real-time demand which could potentially reduce the switching losses.

The system state is sampled as $x(k T)$, where $T$ is the sampling period and $k=0,1,2,3 \cdots$. For notational simplicity, $k$ stands for $k T$ in the remaining part of paper.

A state-feedback switching control law $\sigma(*)$ is proposed as

$$
\begin{aligned}
\sigma(x(k))=\arg \min _{(i, j) \in \Xi \times \Xi}\{ & 2 w_{1}\left(\left(x(k)-x_{e}\right)^{T} P_{j}\left(A_{i} x(k)+B_{i}\right)\right) \\
& \left.+2 w_{2}|i-\sigma(x(k-1))|\right\}
\end{aligned}
$$

where $x_{e}=\left[i_{e}, v_{e}\right]^{T}$ is the reference point obtained by (3); $\Xi=$ $\{1,2\}, w_{1}, w_{2}$ are two positive weighting factors for adjustment of switching frequency; $P_{j} \in \mathbb{R}^{2 \times 2}$ are parameter matrices to be determined later. It should be mentioned here that the control design is based on the Lyapunov stability theorem [30]. 
The switching control law $\sigma(*)$ should firstly guarantees the system stability by finding fastest converging direction of the Lyapunov functional. This feature is fulfilled by the first part of the switching function $\left(\left(x(k)-x_{e}\right)^{T} P_{j}\left(A_{i} x(k)+B_{i}\right)\right)$, which is the derivative of the Lyapunov functional. The second part of the switching law $|i-\sigma(x(k-1))|$ penalizes the switching action. In this way the switching frequency is adjusted by tuning the weighting factor $w_{2}$. The two weighting factor $w_{1}$, $w_{2}$ are utilized to make a trade off between the transient performance and the switching frequency adjustment. The following theorem with proof in Appendix A states that under some conditions the proposed switching control law will drive the system to the neighborhood of the desirable reference points. The proof of the theorem 1 can be found .

Theorem 1: For given positive scalars $\xi, \rho, \varepsilon, \beta, \kappa$, a Lyapunov-Krasovskii functional candidate is defined as

$$
V(\phi(t), t)=V_{1}(\phi(t))+V_{2}(\phi(t), t),
$$

where $\phi(t)=x(t)-x_{e}$, and

$$
\begin{aligned}
V_{1}(\phi(t)) & =\min _{j \in \Xi}\left\{\phi^{T}(t) P_{j} \phi(t)\right\} \\
V_{2}(\phi(t), t) & =(T-\tau) \int_{t-\tau}^{t} e^{\xi(s-t)} \dot{\phi}^{T}(s) Q_{\sigma} \dot{\phi}(s) \mathrm{d} s \\
\tau & =t-t_{k}, t \in[k, k+1) .
\end{aligned}
$$

If the following condition is satisfied

$$
\begin{array}{r}
\dot{V}(\phi(t), t)+\xi V(\phi(t), t)<\rho T+\kappa\left\|x_{e}\right\|_{2}+(\varepsilon+2 \beta) \frac{w_{2}}{w_{1}}, \\
\forall t \in[k, k+1),
\end{array}
$$

then under the switching signal $\sigma(x(k))$ the state of the DCDC converters $x(t)$ will converge to a finite region $\Theta$ around the reference point $x_{e}$, where

$$
\begin{aligned}
\Theta \triangleq\left\{x(t) \in \mathbb{R}^{2}: \min _{j \in \Xi}\left\{\operatorname{eig}_{\min }\left(P_{j}\right)\right\}\left\|x-x_{e}\right\|_{2}\right. \\
\left.\leq \frac{T \rho+\kappa\left\|x_{e}\right\|^{2}}{\xi}+\frac{\varepsilon+2 \beta}{\xi} \frac{w_{2}}{w_{1}}\right\},
\end{aligned}
$$

Therefore, if the system is practically stable under proposed switching signal $\sigma(x(k))$, the inductor current $i_{L}$ and capacitor voltage $v_{C}$ will be stabilized around the desired DC steady state $i_{e}$ and $v_{e}$ respectively with bounded ripples. Furthermore, The boundary of the current ripple and voltage ripple are defined by the region $\Theta$.

Remark 1: The minimum function $V_{1}(\phi(t))$ ensures that the abstract energy associate with the system will not increase at the switching instant. To guarantee that the energy will decrease along the trajectory of the system, it suffices to require that the Lypuanov function does not increase during the sampling interval $[k, k+1)$. A discontinuous term $V_{2}(\phi(t), t)$ is added to achieve this.

We are now in a position to present the main result of the paper. The details of how to solve the unknown Lyapunov matrices $P_{j}$, with which the practical stability condition (8) is satisfied, is demonstrated in the form of Bilinear Matrix Inequalities (BMIs). The explicit proof of Theorem 1 can be found in Appendix B.

Theorem 2: Consider the switched model (1) with sampling period $T$ and given parameters $\xi>0, \rho>0, \varepsilon>0, \kappa>0, w_{1}>$
$0, w_{2} \geq 0$, for a specific reference point $x_{e}$ with corresponding $\lambda \in[0,1]$, if there exist matrices $P_{j}>0, Q_{i}>0$, scalars $\beta>0$, $\mu_{j r}>0$, for $\forall(i, j) \in \Xi \times \Xi, r \in \Xi$ such that

$$
\Psi(0,0, \lambda)<0, \quad \Psi\left(T, T^{2}, \lambda\right)<0,
$$

where

$$
\begin{aligned}
& \Psi\left(\tau, \tau^{2}, \lambda\right) \\
& =\Pi(\tau)+\beta\left(\Omega\left(\tau, \tau^{2}\right)+\Omega\left(\tau, \tau^{2}, \lambda\right)\right)-\sum_{r}^{\Xi} \mu_{j r} \Upsilon_{j}\left(\tau, \tau^{2}\right), \\
& \Pi(\tau)=\left[\begin{array}{cccc}
\Pi(\tau)_{11} & \Pi(\tau)_{12} & 0 & \Pi(\tau)_{14} \\
* & \Pi(\tau)_{22} & 0 & \Pi(\tau)_{24} \\
* & * & -\tau Q_{i} e^{-\xi T} & 0 \\
* & * & * & \Pi(\tau)_{44}
\end{array}\right],
\end{aligned}
$$

$$
\begin{aligned}
& \Pi(\tau)_{11}=A_{i}^{T} P_{j}+P_{j} A_{i}+\xi P_{j}+(T-\tau) A_{i}^{T} Q_{i} A_{i}, \\
& \Pi(\tau)_{12}=(T-\tau) A_{i}^{T} Q_{i} A_{i}+P_{j} A_{i}, \\
& \Pi(\tau)_{14}=(T-\tau) A_{i}^{T} Q_{i} B_{i}+P_{j} B_{i}, \\
& \Pi(\tau)_{22}=(T-\tau) A_{i}^{T} Q_{i} A_{i}-\kappa, \\
& \Pi(\tau)_{24}=(T-\tau) A_{i}^{T} Q_{i} B_{i}, \\
& \Pi(\tau)_{44}=(T-\tau) B_{i}^{T} Q_{i} B_{i}-\rho T-(\varepsilon+2 \beta) \frac{w_{2}}{w_{1}} \\
& \Omega\left(\tau, \tau^{2}\right)= \\
& {\left[\begin{array}{cccc}
-A_{i}^{T} P_{j}-P_{j} A_{i} & -P_{i} A_{i} & \tau\left(A_{i}^{T} P_{j}+P_{j} A_{i}\right) & -P_{j} B_{i} \\
* & 0 & \tau A_{i}^{T} P_{j} & 0 \\
* & * & -\tau^{2}\left(A_{i}^{T} P_{j}+P_{j} A_{i}\right) & \tau P_{i} B_{i} \\
* & * & * & 2 \frac{w_{2}}{w_{1}} \mathbf{I}
\end{array}\right],} \\
& \Omega\left(\tau, \tau^{2}, \lambda\right)= \\
& {\left[\begin{array}{cccc}
A^{T}(\lambda) P_{j}+P_{j} A(\lambda) & 0 & -\tau\left(A^{T}(\lambda) P_{j}+P_{j} A(\lambda)\right) & 0 \\
* & 0 & 0 & 0 \\
* & * & \tau^{2}\left(A^{T}(\lambda) P_{j}+P_{j} A(\lambda)\right) & 0 \\
* & * & * & 0
\end{array}\right],} \\
& \Upsilon_{j}\left(\tau, \tau^{2}\right)=\left[\begin{array}{cccc}
P_{j}-P_{r} & 0 & -\tau\left(P_{j}-P_{r}\right) & 0 \\
* & 0 & 0 & 0 \\
* & * & \tau^{2}\left(P_{j}-P_{r}\right) & 0 \\
* & * & * & 0
\end{array}\right]
\end{aligned}
$$

the condition (8) is fulfilled. Then the DC-DC converter is practically stabilizable to the region $\Theta$ around the desired reference point $x_{e}$ under the switching signal (4).

Remark 2: The tightness of the region (9) is proportional to the sampling period $T$. Thus, for other fixed parameters, a higher sampling rate will result in a tighter boundary, which means a smaller converter output voltage ripple.

It should be mentioned that unlike the traditional PWM control which has a fixed switching frequency, the switching frequency is unfixed for the proposed method. As the switching signal keeps constant during each sampling cycle, the average switching frequency of proposed method is bounded by the sampling frequency which is more applicable in practice than the other similar stability based methods [31] with the assumption of infinite switching frequency. It is obvious that the switching frequency is inversely proportional to the ripple. Hence, it is crucial to reach a compromise between ripple 
minimization and switching frequency reduction because overrapid switching will reduce the energy efficiency of the converter and pose a challenge to thermal management.

In the proposed switching signal function

$$
\begin{gathered}
\sigma(x(k))=\arg \min _{(i, j) \in \Xi \times \Xi}\left\{2 w_{1}\left(\left(x(k)-x_{e}\right)^{T} P_{j}\left(A_{i} x(k)+B_{i}\right)\right)\right. \\
+2 w_{2} \mid i-\sigma(x(k-1) \mid\},
\end{gathered}
$$

the weighting factor $w_{1}$ and $w_{2}$ are introduced to adjust the average switching frequency. For the term $\mid i-\sigma(x(k-1) \mid$, if $i$ is equal to the previous switching state $\sigma(x(k-1)$, it will be zero; otherwise it will be one. Due to the min property of the switching signal function, it will prevent the change of the switch state if the practical stability can be guaranteed. By this way, some unnecessary switching can be avoided.

The region $\Theta$ is proportional to the weighing factor $w_{2}$ for a fixed $w_{1}$. Hence, if $w_{2}$ is increasing, the region become larger which means larger current and voltage ripples. In the meantime, the average switching frequency is decreased. One may argue that the change of the factor $w_{2}$ will result in the invalidity of inequalities (10). In the following corollary, the practical stability holds when the weighting factor $w_{2}$ changes. The proof is presented in Appendix C.

Corollary 1: Consider the switched model (1) with sampling period $T$ and parameters $\xi>0, \rho>0, \varepsilon>0, \kappa>0$ for a specific reference point $x_{e}$ with corresponding $\lambda \in[0,1]$. If there exist matrices $P_{j}>0, Q_{i}>0$, scalars $\beta>0, \mu_{j r}>0$, for $\forall(i, j) \in \Xi \times \Xi, r \in \Xi$ such that

$$
\Psi(0,0, \lambda)<0, \quad \Psi\left(T, T^{2}, \lambda\right)<0,
$$

with a given $w_{1}>0$ and $w_{2}=0$, then the condition (8) is fulfilled and the DC-DC converter is practically stabilizable to the region $\Theta$ around the desired reference point $x_{e}$ under the switching signal

$$
\begin{aligned}
\sigma(x(k))=\arg \min _{(i, j) \in \Xi \times \Xi}\{ & 2 w_{1}\left(\left(x(k)-x_{e}\right)^{T} P_{j}\left(A_{i} x(k)+B_{i}\right)\right) \\
& +2 w_{2} \mid i-\sigma(x(k-1) \mid\},
\end{aligned}
$$

with the same $w_{1}>0$ and any $w_{2} \geq 0$.

Remark 3: According to Corollary 1, the weighting factor $w_{2}$ can be adjusted online without re-design the switching function $\sigma(x(k))$. The benefit is that the balance between ripple minimization and switching frequency reduction can be adapted depending on the practical situation in real-time.

Remark 4: Even though the practical stability preserves theoretically for any $w_{2}>0$, one should be careful when increasing the weighting $w_{2}$. A very large $w_{2}$ will result in very large current and voltage ripples. In such a situation, the DC-DC converter is practically unstable.

Remark 5: For simplicity, in this paper, we assume there is no delay of measuring the system state. However, in practice if the sampling of the state is slow and delayed, it might be troublesome due to measurements are mismatched. This issue may be solved by introducing the predictive technique for the system state [32].

Until now, the proposed method is design for a specific reference point $x_{e}$. As we mentioned before, in some applications, the output voltage of DC-DC power converter are required to track a changing reference signal or adjust manually. In the following corollary, the proposed control scheme is extended to a range of reference points. Therefore, the switching signal function can drive the state of DC-DC converter tracking different reference points within the range in real-time without re-design. The proof of the corollary is given in Appendix D.

Corollary 2: Consider the switched model (1) with sampling period $T$ and given parameters $\xi>0, \rho>0, \varepsilon>0, \kappa>0$, $0<\lambda_{\text {min }}<\lambda_{\text {max }}<1$. If there exist matrices $P_{j}>0, Q_{i}>0$, scalars $\beta>0, \mu_{j r}>0$, for $\forall(i, j) \in \Xi \times \Xi, r \in \Xi$ such that

$$
\begin{aligned}
& \Psi\left(0,0, \lambda_{\text {min }}\right)<0, \quad \Psi\left(0,0, \lambda_{\max }\right)<0, \\
& \Psi\left(T, T^{2}, \lambda_{\text {min }}\right)<0, \quad \Psi\left(T, T^{2}, \lambda_{\text {max }}\right)<0,
\end{aligned}
$$

with a given $w_{1}>0$ and $w_{2}=0$, then the condition (8) is fulfilled and the DC-DC converter is practical stabilizable to the region $\Theta$ around any desired reference point $x_{e}$ with corresponding $\lambda \in\left[\lambda_{\min }, \lambda_{\max }\right]$ under the switching signal

$$
\begin{gathered}
\sigma(x(k))=\arg \min _{(i, j) \in \Xi \times \Xi}\left\{2 w_{1}\left(\left(x(k)-x_{e}\right)^{T} P_{j}\left(A_{i} x(k)+B_{i}\right)\right)\right. \\
+2 w_{2} \mid i-\sigma(x(k-1) \mid\}
\end{gathered}
$$

with the same $w_{1}>0$ and any $w_{2} \geq 0$.

In the previous parts, it is assumed that the parameters of DC-DC buck converters are accurate and invariant. However, in practice this is hard to guaranteed, especially for the load $R$. This issue could be addressed by modifying the switched model (1) as follow to include the parameter uncertainty.

$$
\begin{array}{r}
\dot{x}(t)=\left(A_{\sigma}+\sum_{m=1}^{q} \delta_{\sigma}^{m} \hat{A}_{\sigma}^{m}\right) x(t)+\left(B_{\sigma}+\sum_{m=1}^{q} \delta_{\sigma}^{m} \hat{B}_{\sigma}^{m}\right), \\
\sigma \in \Xi \triangleq\{1,2\}, \delta_{\sigma}^{m}>0, \sum_{m=1}^{q} \delta_{\sigma}^{m}=1, m \in N^{+},
\end{array}
$$

$A_{\sigma}$ and $B_{\sigma}$ are system matrices with nominal parameters, while $\hat{A}_{\sigma}^{m}$ and $\hat{A}_{\sigma}^{m}$ represent the vertices of the perturbations.

For examples, if we assume the load $R$ varies between $R_{\text {min }}$ and $R_{\max }$ with nominal value $R_{N}$. The perturbations matrices should be calculated as follow:

$$
\begin{array}{ll}
\hat{A}_{1}^{1}=\hat{A}_{2}^{1}=\left[\begin{array}{lc}
0 & 0 \\
0 & \frac{R_{\max }-R_{N}}{R_{N} C R_{\max }}
\end{array}\right] & \hat{A}_{1}^{2}=\hat{A}_{2}^{2}=\left[\begin{array}{cc}
0 & 0 \\
0 & \frac{R_{\min }-R_{N}}{R_{N} C R_{\min }}
\end{array}\right] \\
\hat{B}_{1}^{1}=\hat{B}_{2}^{1}=\left[\begin{array}{l}
0 \\
0
\end{array}\right] & \hat{B}_{1}^{2}=\hat{B}_{2}^{2}=\left[\begin{array}{l}
0 \\
0
\end{array}\right]
\end{array}
$$

For simplicity, here we assume the switching law is based on the continuous state and without switching frequency adjustment.

$$
\sigma(x(k))=\arg \min _{(i, j) \in \Xi \times \Xi}\left\{2\left(\left(x(t)-x_{e}\right)^{T} P_{j}\left(A_{i} x(t)+B_{i}\right)\right)\right\}
$$

The following theorem states that under some BMI conditions, the switching control law (14) will drive the system to the neighborhood of the desirable equilibrium (3). The proof of the theorem is given in Appendix E.

Theorem 3: Consider the switched model (13) with given parameters $\xi>0$, for a specific reference point $x_{e}$ with 
corresponding $\lambda \in[0,1]$, if there exist matrices $P_{j}>0$, scalars $\beta>0$, for $\forall(i, j) \in \Xi \times \Xi$, such that

$$
\left[\begin{array}{cc}
\left(A(\lambda)+\hat{A}_{i}^{m}\right)^{T} P_{j}+P_{j}\left(A(\lambda)+\hat{A}_{i}^{m}\right)+\xi P_{j} & P_{j} \hat{B}_{i}^{m} \\
* & -\beta
\end{array}\right]<0,
$$

the condition

$$
\dot{V}_{1}(\phi(t), t)+\xi V_{1}(\phi(t), t)<\beta .
$$

is fulfilled. Then the DC-DC converter is practically stabilizable to the region $\hat{\Theta} \triangleq\left\{x(t) \in \mathbb{R}^{2}: \min _{j \in \Xi}\left\{\operatorname{eig}_{\min }\left(P_{j}\right)\right\}\left\|x-x_{e}\right\|_{2} \leq\right.$ $\left.\frac{2 \beta}{\xi}\right\}$, around the desired reference point $x_{e}$ under the switching signal (14).

One drawback of the proposed control techniques is the dependence on correct model parameters. In the case of load varying, for example, errors may be inferred in the steady state output voltage. To minimize this error, a common practice is to introduce a outer integration loop to correct the reference points $x_{e}$ by adjusting the $\lambda$ as follow:

$$
\lambda_{c}=\lambda-\sum_{j=1}^{k} \alpha\left(v_{C, j}-v_{C, e}\right)
$$

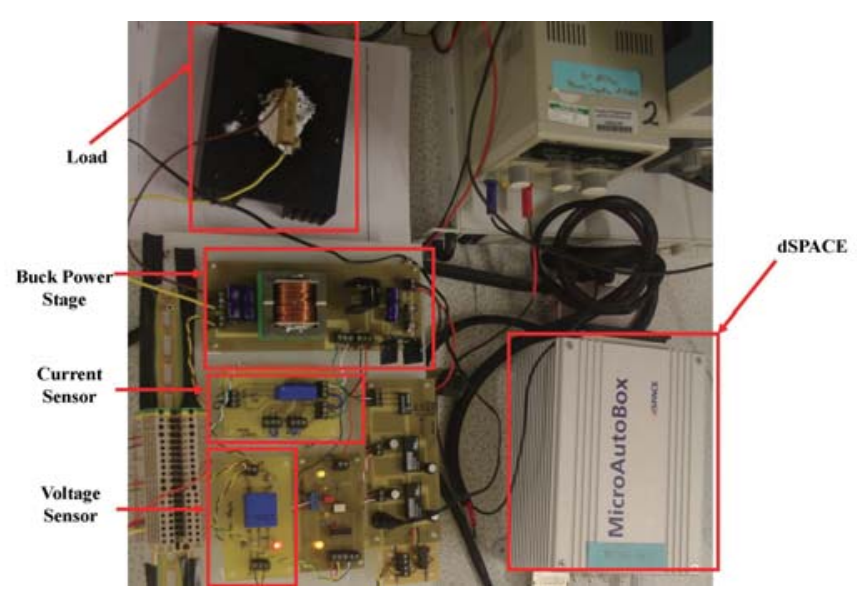

Figure 2: The laboratory prototype of a DC-DC buck converter.

\section{Practical application to DC-DC Buck CONVERTERS}

\section{A. Experimental Setup}

In order to test the effectiveness of the proposed control algorithm, the switching controller is applied on a DC-DC buck converter as shown in Fig. 1. A laboratory prototype has been designed based on the conventional design procedure in [33] as shown in Fig. 2. The parameters of the buck stage are $L=616.3 \mu H, C=880 \mu F, R=4.90 \Omega$ (CCM load), $R=30 \Omega$ (DCM load), $V_{g}=20 \mathrm{~V}$. The test platform consist of a dSPACE MicroAutoBox(1401/1511) 2nd generation with built-in IBM PPC 750GL, $900 \mathrm{MHZ}$ processor, a current sensor board with current transducer $L A H 25-N P$ and a voltage sensor board with voltage transducer $L V 25-P$ both connected to separate ADC channels of the dSPACE. A MOSFET drive board is also designed to convert the switching signal from
dSPACE to MOS level and isolate the buck converter and dSPACE for safety. The proposed algorithms are implemented on the dSPACE. The experimental data is automatically saved by the dSPACE control software ControlDesk and then read offline by Matlab.

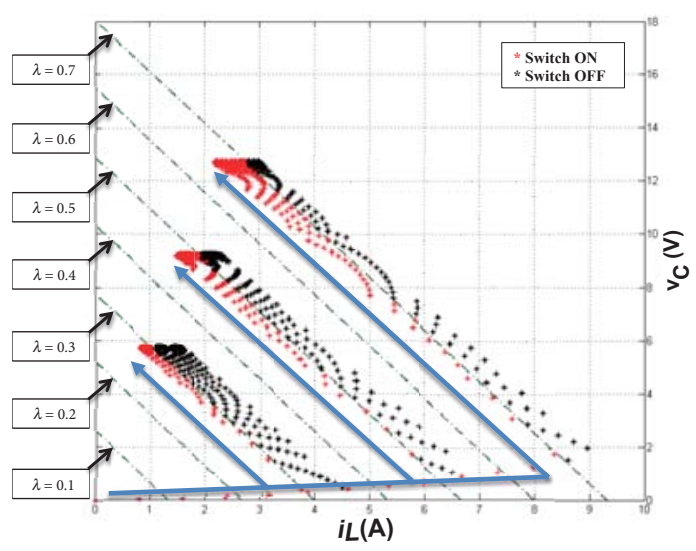

Figure 3: Switching surface for different reference points.

\section{B. Switching signal function analysis}

Although the stability condition for the switching signal function design seems complicated, the precalculation of the parameters makes it very simple to implement. The nature of the switching signal function $\sigma(x(k))$ is to partition the state space into different regions. After searching the state space, the switching surface for different reference points can be calculated. The state space partition can be seen in Fig. 3. For the Buck converter, $A_{1}$ and $A_{2}$ are equal and the matrices $P_{1}, P_{2}$ are similar. Hence, the switching surfaces are almost linear as seen in Fig. 3, which are presented by the dashed lines. The switching signal is defined as $O N$ if the system state is recognized in the left of the selected switching surface, otherwise the switching signal is defined as $O F F$. In Fig. 3, the transition behaviour of the system state with three different reference points is presented as examples. The colours of asterisks mean different switching signals, that is: red means $O N$, black means $O F F$; and the arrows indicate the time revolution of the system state $x(t)$. As one can seen, if the state is away from switching surface, the switching signal will drive the state towards the switching surface. If the state is around the switching surface, then it will be driven to the origin along the surface. Finally, the system state $x(t)$ will be stabilized around the desired reference point.

\section{Implementation Procedure}

The proposed control scheme could be implemented in two step: offline calculation of the controller parameters and the online implementation. In the following algorithm, the step-bystep instruction is presented about how to solve the controller based on Corollary 2. After obtaining the required controller parameters $P_{j}$, the online implementation could follow the flow chart in Fig. 4. 


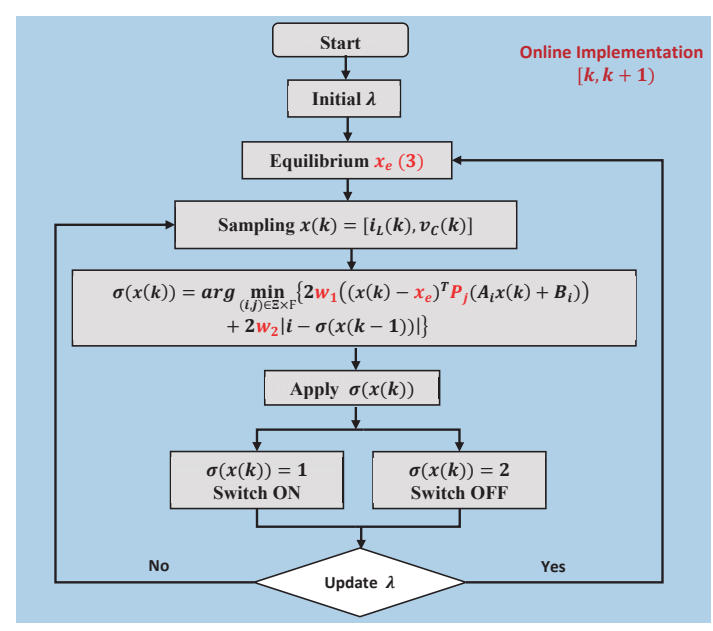

Figure 4: Flow chart of the online implementation.

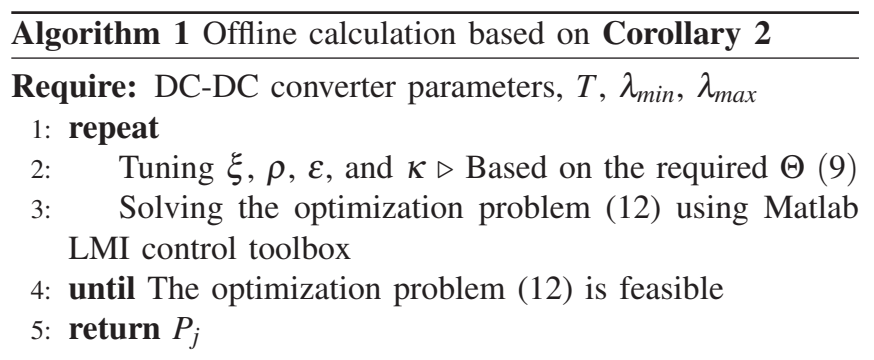

\section{Continuous Conduction Mode (CCM)}

In this mode, the load is set as $4.90 \Omega$.

a) Start-up response under different sampling frequency: In this part, the switching controller in Corollary 2 are solved for different sampling frequency. The tuning parameters are given as: $\xi=2 \times 10^{-4}, \rho=1 \times 10^{-4}, \varepsilon=2, \kappa=1 \times 10^{-4}$. The conditions (2) are feasible for different sampling frequency: $f_{s}=10,20,40 \mathrm{kHz}$. Figs. 5-7 show the experimental behavior of the system state under different sampling frequencies. The averaged switching frequency $f_{a}$ is also presented in each scenarios. One can clearly observe that the proposed methods can be applicable to a wide sampling frequency range and generate similar transition performance as shown in simulation results.

b) Reference tracking: As we claimed in Corollary 2, the proposed switching controller is solved for $\forall \lambda \in[0,1]$, which means that the switching controller is able to drive the converter to track different reference point $x_{e}$. In the part, the sampling frequency is $10 \mathrm{kHz}$ with parameters $\xi=2 \times 10^{-4}$, $\rho=1 \times 10^{-4}, \varepsilon=2, \kappa=1 \times 10^{-4}$. As pointed out previously, the controller is robust to the change of the reference points as shown in Fig. 8. Both the capacitor voltage and the inductor current have been controlled to track the corresponding reference effectively.

c) Switching frequency tuning: As we proposed, the average switching frequency can be optimized by tuning a set of weighting factors. It gives more flexility for the balance between the voltage ripple and switching frequency. This is also verified in the real experiment. A high sampling frequency $40 \mathrm{kHz}$ is used to solve the switching controller in (a) $\mathrm{v}_{\mathrm{C}}$

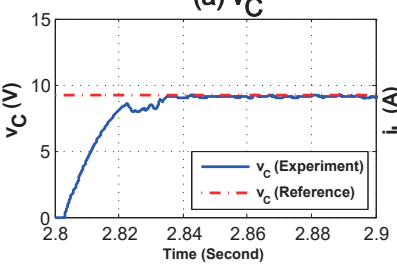

(c) $\mathrm{v}_{\mathrm{C}}$ (Zoomed)

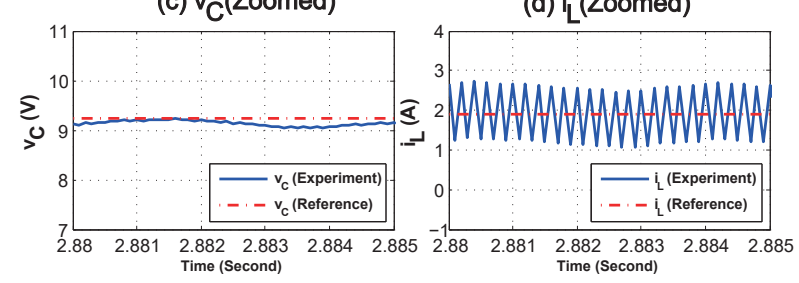

(b) $\mathrm{i}_{\mathrm{L}}$

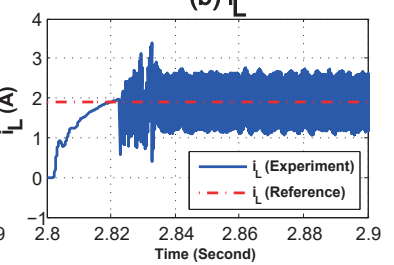

(d) $\mathrm{i}_{\mathrm{L}}$ (Zoomed)
Figure 5: The response of $v_{C}$ and $i_{L}\left(f_{s}=10 \mathrm{kHz}, f_{a}=5.1\right.$ $k H z, \mathrm{CCM})$ (a) $v_{C}$

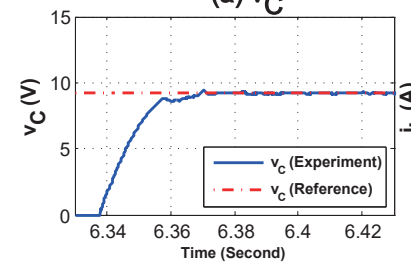

(c) $\mathrm{v}_{\mathrm{C}}$ (Zoomed)

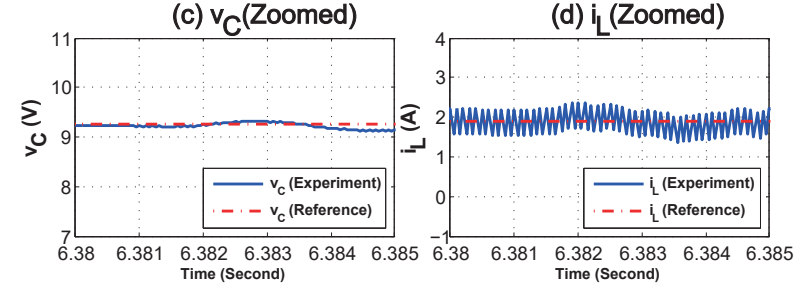

(b) $i_{L}$

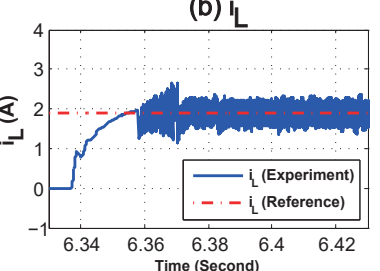

(d) $\mathrm{i}_{\mathrm{L}}$ (Zoomed)

Time (Second)
Figure 6: The response of $v_{C}$ and $i_{L}\left(f_{s}=20 \mathrm{kHz}, f_{a}=10\right.$ $\mathrm{kHz}, \mathrm{CCM})$

Corollary 2 with parameters $\xi=2 \times 10^{-4}, \rho=1 \times 10^{-4}, \varepsilon=2$, $\kappa=1 \times 10^{-4}$. The result is shown in Fig. 9. As we can see, as $w_{2}$ increases, the average switching frequency decreases rapidly but the voltage ripple just increases slightly.

d) Load variation (Simulation Results): In this part, simulation work has been carried out to verify the switching law (14) when the load $R$ is varying. The sampling frequency is set to $20 \mathrm{kHz}$. The BMI (15) is solved offline with $R_{\max }=10 \Omega$ and $R_{\text {min }}=4.9 \Omega$. The tuning parameter $\alpha$ of the outer integration loop is set to $1 \times 10^{-3}$. Hence, the switching law should work when the load is varying between $R_{\min }$ and $R_{\max }$. In Fig. 10, the worst scenario is given when the load step up to $10 \Omega$ from the nominal value $4.9 \Omega$ at $0.01 s$ and step back to nominal value at $0.02 s$. As we can see from Fig. 10, during $0.01 s$ to $0.02 s$, the voltage follows the reference without steady state error, while the current does not due to load variation.

e) Comparison with PI Controller (Simulation Results): In this part, the proposed switching control approach is further compared with a traditional industry-standard PI-type controller tuned on the basis of a linearized averaged model 
(a) $v_{C}$

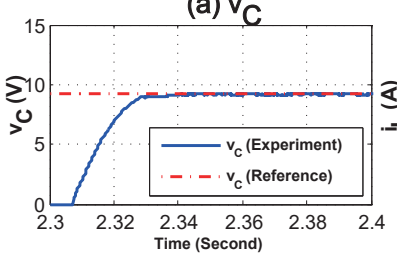

(c) $\mathrm{v}_{\mathrm{C}}$ (Zoomed)

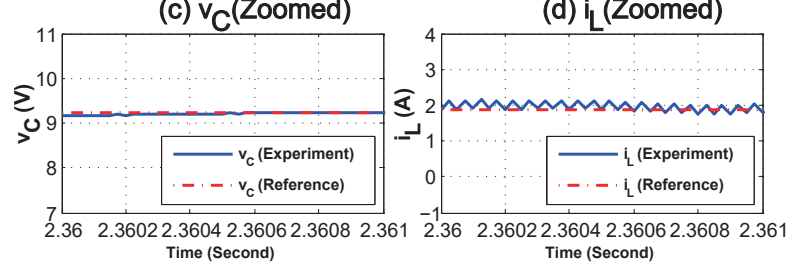

Figure 7: The response of $v_{C}$ and $i_{L}\left(f_{s}=40 \mathrm{kHz}, f_{a}=20.3\right.$ $k H z, \mathrm{CCM})$

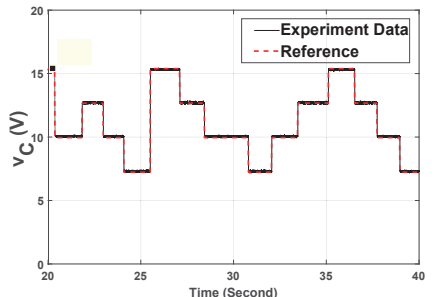

(a) $v_{C}$

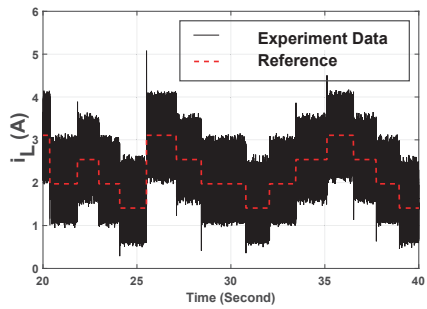

(b) $i_{L}$
Figure 8: The response of $v_{C}$ and $i_{L}$ to different reference points (CCM mode).

(averaged control scheme) [34]. The sampling frequency of the proposed method and the switching frequency of PWM signal are both set to $20 \mathrm{kHz}$. And the weighting factor $w_{2}$ is set to 0 for a fair comparison. The output voltage and inductor current responses under these two control scheme are shown in Fig. 11. obviously, the proposed method presented better transient performance than the PI controller for both the voltage and current response.

The phase diagrams for both proposed method and PI controller are shown in Fig. 12. As explained in the previous

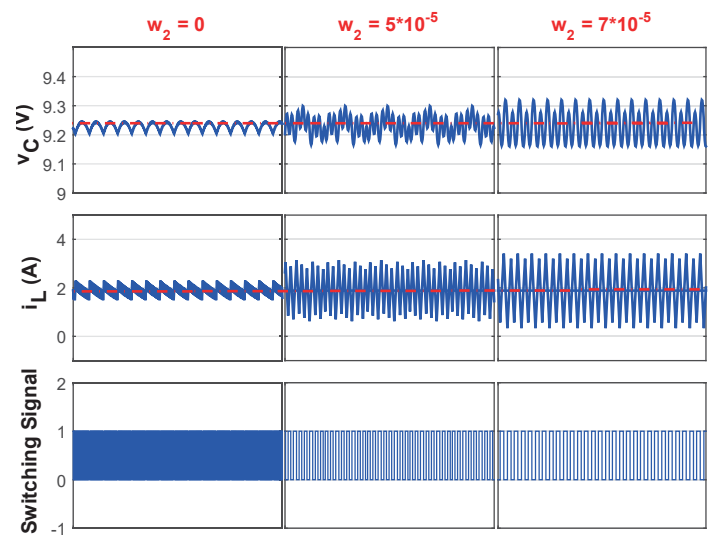

Figure 9: Experimental behaviour of $v_{C}, i_{L}$ and switching signal $\sigma$ when tuning the weighting factor $w_{2}$ (CCM mode).
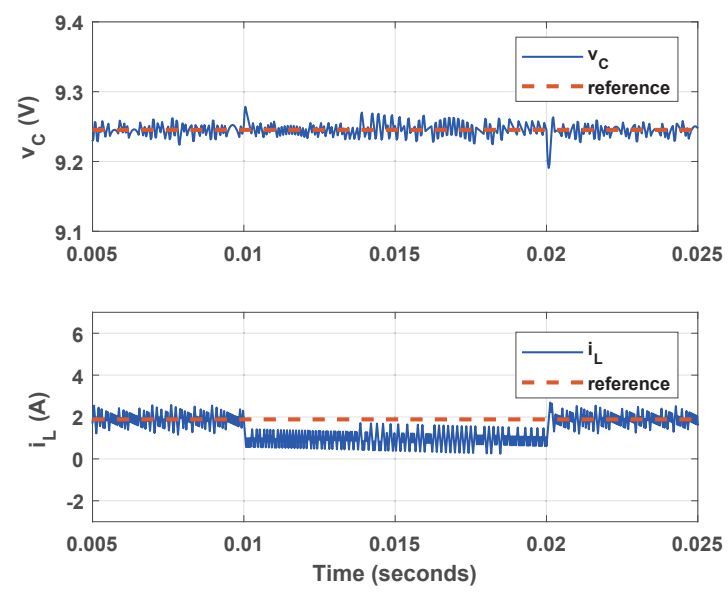

Figure 10: Load variation from $4.9 \Omega$ to $10 \Omega$ at $0.01 s$ and back to $4.9 \Omega$ at $0.02 \mathrm{~s}$

subsection B and Theorem 1, the proposed control method defines a switching surface Fig.3 and a converged region (9). Hence, the designed controller will drive the system state to the switching surface first and to the converged region along the switching surface. In addition, as the controller will choose the switching action which is able to drive the system state along the fastest possible converging direction. All these characteristic will result in a trajectory with shorter transition period and no/smaller state overshoot for both current and voltage state.

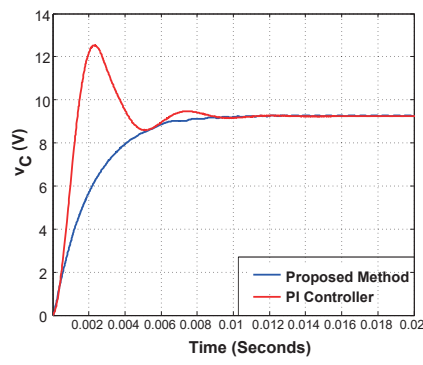

(a) $v_{C}$

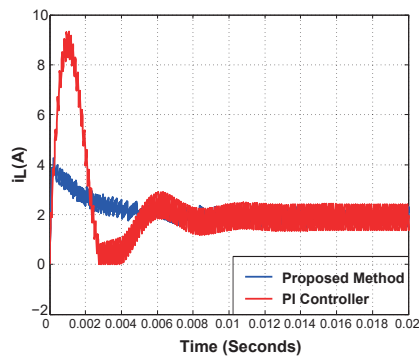

(b) $i_{L}$
Figure 11: The responses of $v_{C}$ and $i_{L}$ under proposed method and a PI controller (Simulation).

\section{E. Discontinuous Conduction Mode (DCM)}

In this mode, the load is set as $30 \Omega$ and sampling frequency is fixed as $40 \mathrm{kHz}$. The parameters that is used to solve the switching controller $\sigma(x(k))$ in Corollary 2 are $\xi=2 \times 10^{-3}$, $\rho=1 \times 10^{-4}, \varepsilon=2, \kappa=1 \times 10^{-4}$. The start-up response is shown in Fig. 13. One can easily notice that the inductor current $i_{L}$ is discontinues. Furthermore, the converter is also controlled to track different reference points by the proposed switching controller as depicted in Fig. 14. From Fig. 15, it can be seen that when tuning the weighting factor $w_{2}$, the average switching frequency presents similar behaviour as the CCM mode. 


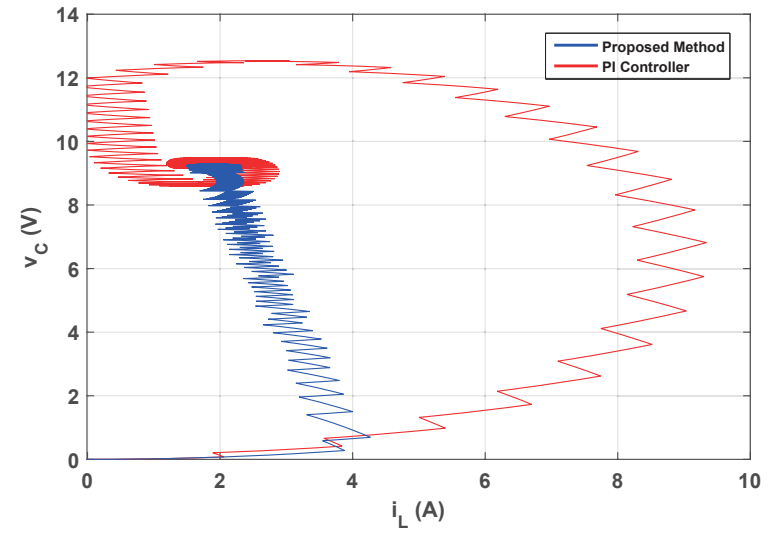

Figure 12: Phase diagrams of the proposed method and a PI controller.
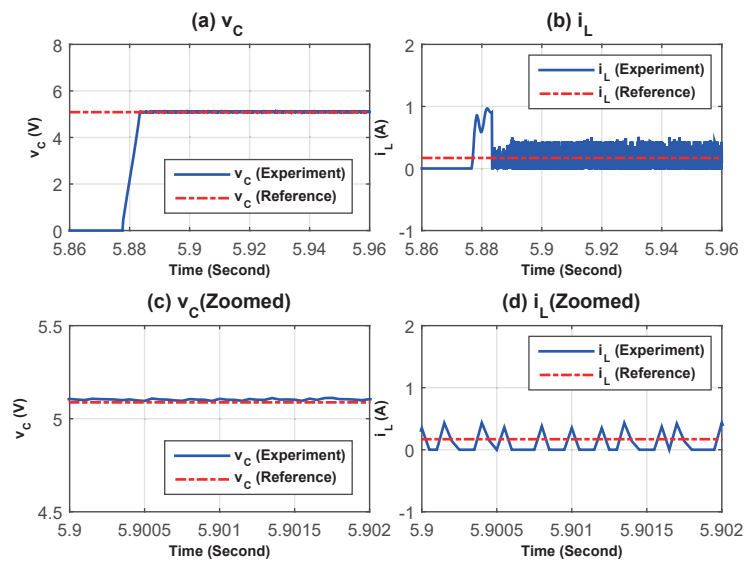

Figure 13: The start-up response of $v_{C}$ and $i_{L}$ (DCM mode)

\section{CONCLUSIONS}

We have proposed a novel switching controller for DC-DC converters. The converters have been modeled as sampled-data switched systems. A composite Lyapunov functional has been used to design the switching signal function with guaranteed stability and tracking property. Furthermore, the switching frequency can be adjusted in real-time with guaranteed stability. The future work may include developing different Lyapunov functional to compare the conservatism, extending the results to more complicated power electronic converters, such as

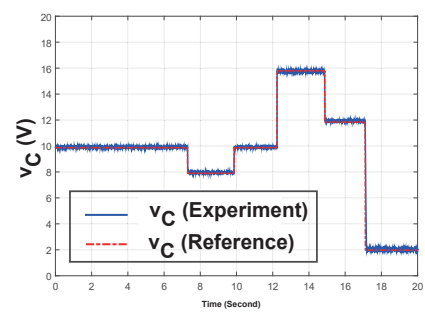

(a) $v_{C}$

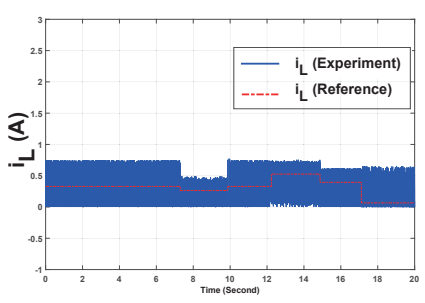

(b) $i_{L}$
Figure 14: The response of $v_{C}$ and $i_{L}$ to different reference points (DCM mode).

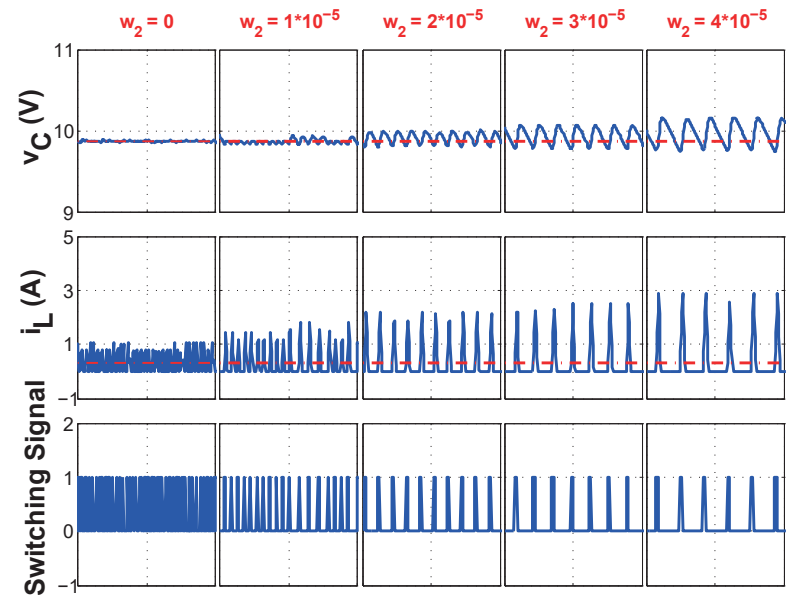

Figure 15: Experimental behaviour of $v_{C}, i_{L}$ and switching signal $\sigma$ when tuning the weighting factor $w_{2}$ (DCM mode).

multilevel inverters, bidirectional converters, and improve the robustness of the closed-loop system.

\section{ApPendix A: Proof of TheOREM 1}

Proof: We note from the min property of $V_{1}(\phi(t))$ that at the switching instant $k$,

$$
\begin{aligned}
V_{1}\left(\phi(k), t_{k}^{+}\right) & =\phi^{T}(k) P_{j\left(\sigma_{k}\right)} \phi(k) \\
& \leq \phi^{T}(k) P_{j\left(\sigma_{k-1}\right)} \phi(k)=V_{1}\left(\phi(k), t_{k}^{-}\right)
\end{aligned}
$$

which means that the Lyapunov functional $V_{1}(e(t))$ does not grow after each switching instant. By using the comparison principle, we have that if (8) holds, then

$$
\begin{aligned}
V_{1}(\phi(t), t) \leq & e^{-2 \xi\left(t-t_{k}\right)} V_{1}\left(\phi(k), t_{k}^{+}\right)+\theta \int_{t_{k}}^{t_{k+1}} e^{-2 \xi(t-s)} d s \\
\leq & e^{-2 \xi\left(t-t_{k-1}\right)} V_{1}\left(\phi(k-1), t_{k-1}^{+}\right) \\
& +\theta \int_{t_{k-1}}^{t} e^{-2 \xi(t-s)} d s \\
\leq & \ldots \leq e^{-2 \xi t} V_{1}(\phi(0), 0)+\theta \int_{0}^{t} e^{-2 \xi(t-s)} d s
\end{aligned}
$$

where

$$
\theta=\rho T+\kappa\left\|x_{e}\right\|_{2}+(\varepsilon+2 \beta) \frac{w_{2}}{w_{1}},
$$

which means that for $t \rightarrow \infty, x(t)$ exponentially converges to the region $\Theta$.

\section{Appendix B: Proof of TheOREM 2}

Proof: Without loss of generality, we assume that at time $k, \sigma(x(k))=i$ and $V_{1}(\phi(k))=\min _{j \in \Xi}\left\{\phi(k)^{T} P_{j} \phi(k)\right\}=$ $\phi(k)^{T} P_{j} \phi(k)$. Then along the solution of (1), the derivative of the chosen Lyapunov functional (6) can be calculated:

$$
\begin{aligned}
\dot{V}_{1} & =\min \left\{\dot{\phi}^{T}(t) P_{j} \phi(t)+\phi^{T}(t) P_{j} \dot{\phi}(t)\right\} \\
& =\min \left\{2 \phi^{T}(t) P_{j}\left(A_{i} \phi(t)+A_{i} x_{e}+B_{i}\right)\right\} \\
& \leq \phi^{T}(t)\left(A_{i}^{T} P_{j}+P_{j} A_{i}\right) \phi(t)+2 \phi(t) P_{j}\left(A_{i} x_{e}+B_{i}\right) .
\end{aligned}
$$


Applying Jensen's inequality [35] yields,

$$
\begin{aligned}
\dot{V}_{2}+\xi V_{2} \leq & (T-\tau)\left(A_{i} \phi(t)+A_{i} x_{e}+B_{i}\right)^{T} Q_{i}\left(A_{i} \phi(t)+A_{i} x_{e}+B_{i}\right) \\
& -\tau v^{T}(t) Q_{i} v(t) e^{-\xi T},
\end{aligned}
$$

where $v(t)=\frac{x(t)-x\left(t_{k}\right)}{\tau}, \tau=t-t_{k}$.

Define $\eta(t)=\left[\begin{array}{llll}\phi(t) & x_{e} & v(t) & 1\end{array}\right]^{T}$, the condition (8) holds if

$$
\eta^{T}(t) \Pi(\tau) \eta(t)<0 .
$$

Based on the proposed switching signal function (4), for $\forall l \in \Xi$, the following inequality holds:

$$
\begin{gathered}
2 w_{1} \phi(k)^{T} P_{j}\left(A_{l} \phi(k)+A_{l} x_{e}+B_{l}\right)+2 w_{2}\left|l-\sigma_{k-1}\right| \geq \\
\quad 2 w_{1} \phi(k)^{T} P_{j}\left(A_{i} \phi(k)+A_{i} x_{e}+B_{i}\right)+2 w_{2}\left|i-\sigma_{k-1}\right| .
\end{gathered}
$$

Rearranging the inequality yields,

$$
2 w_{1} \phi(k)^{T} P_{j}\left(A_{l} \phi(k)+A_{l} x_{e}+B_{l}\right)+2 \frac{w_{2}}{w_{1}}\left(\left|l-\sigma_{k-1}\right|-\left|i-\sigma_{k-1}\right|\right)
$$$$
\geq 2 w_{1} \phi(k)^{T} P_{j}\left(A_{i} \phi(k)+A_{i} x_{e}+B_{i}\right) .
$$

Since $-1 \leq\left|l-\sigma_{k-1}\right|-\left|i-\sigma_{k-1}\right| \leq 1, \forall l \in \Xi$, if (23) holds, the following inequality holds:

$$
\begin{aligned}
2 w_{1} \phi(k)^{T} P_{j}\left(A_{l} \phi(k)+A_{l} x_{e}+B_{l}\right)+2 \frac{w_{2}}{w_{1}} \\
\geq 2 w_{1} \phi(k)^{T} P_{j}\left(A_{i} \phi(k)+A_{i} x_{e}+B_{i}\right) .
\end{aligned}
$$

When $l=1$, multiplying the inequality (24) by $\lambda$ yields,

$$
\begin{aligned}
2 w_{1} \phi(k)^{T} P_{j}\left(\lambda A_{1} \phi(k)+\lambda A_{1} x_{e}+\lambda B_{1}\right)+2 \frac{w_{2}}{w_{1}} \\
\geq 2 w_{1} \phi(k)^{T} P_{j}\left(\lambda A_{i} \phi(k)+\lambda A_{i} x_{e}+\lambda B_{i}\right) .
\end{aligned}
$$

When $l=2$, multiplying the inequality $(24)$ by $(1-\lambda)$ yields,

$2 w_{1} \phi(k)^{T} P_{j}\left((1-\lambda) A_{2} \phi(k)+(1-\lambda) A_{2} x_{e}+(1-\lambda) B_{2}\right)+2 \frac{w_{2}}{w_{1}}$
$\geq 2 w_{1} \phi(k)^{T} P_{j}\left((1-\lambda) A_{i} \phi(k)+(1-\lambda) A_{i} x_{e}+(1-\lambda) B_{i}\right)$.

Summing up (25) and (26), the following inequality holds: $2 \phi^{T}(k) P_{j}\left(A(\lambda)-A_{i}\right) \phi(k)-2 \phi^{T}(k) P_{j}\left(A_{i} x_{e}+B_{i}\right)+2 \frac{w_{2}}{w_{1}} \geq 0$,

where the relations $A(\lambda)=\lambda A_{1}+(1-\lambda) A_{2}, B(\lambda)=\lambda B_{1}+$ $(1-\lambda) B_{2}$, and $A(\lambda) x_{e}+B(\lambda)=0$ are used.

For $\phi(k)=\phi(t)-\tau v(t)$, and using the same variable $\eta(t)$, the above inequality becomes:

$$
\eta^{T}(t)\left(\Omega\left(\tau, \tau^{2}\right)+\Omega\left(\tau, \tau^{2}, \lambda\right)\right) \eta(t) \geq 0
$$

Another assumption $V_{1}(\phi(k))=\min _{j \in \Xi}\left\{\phi(k)^{T} P_{j} \phi(k)\right\}=$ $\phi(k)^{T} P_{j} \phi(k)$ is satisfied only if

$$
\phi^{T}(k) P_{j} \phi(k) \leq \phi^{T}(k) P_{r} \phi(k), \forall r \in \Xi
$$

Using the expression $\phi(k)=\phi(t)-\tau \nu(t)$ and the same variable $\eta(t)$, the above inequality becomes:

$$
\eta^{T}(t) \Upsilon_{j}\left(\tau, \tau^{2}\right) \eta(t) \leq 0
$$

Therefore, (8) holds if (21) holds in the condition of (28), (30), that is,

$$
\eta^{T}(t) \Pi(\tau) \eta(t)<0
$$

$$
\begin{array}{ll}
\text { s.t. } & \eta^{T}(t)\left(\Omega\left(\tau, \tau^{2}\right)+\Omega\left(\tau, \tau^{2}, \lambda\right)\right) \eta(t) \geq 0 \\
& \eta^{T}(t) \Upsilon_{j}\left(\tau, \tau^{2}\right) \eta(t) \leq 0 \quad \forall(i, j) \in \Xi \times \Xi
\end{array}
$$

Applying the S-procedure, the above condition holds if $\eta^{T}(t) \Pi(\tau) \eta(t)+\beta \eta^{T}(t)\left(\Omega\left(\tau, \tau^{2}\right)+\Omega\left(\tau, \tau^{2}, \lambda\right)\right) \eta(t)$

$$
\begin{aligned}
& -\sum_{r}^{\Xi} \mu_{j r} \eta^{T}(t) \Upsilon_{j}\left(\tau, \tau^{2}\right) \eta(t)<0 \\
& \exists \beta>0, \exists \mu_{j r}>0, \forall(i, j) \in \Xi \times \Xi .
\end{aligned}
$$

Rearranging the inequality yields,

$$
\Psi\left(\tau, \tau^{2}, \lambda\right)
$$

$$
=\Pi(\tau)+\beta\left(\Omega\left(\tau, \tau^{2}\right)+\Omega\left(\tau, \tau^{2}, \lambda\right)\right)-\sum_{r}^{\Xi} \mu_{j r} \Upsilon_{j}\left(\tau, \tau^{2}\right)<0
$$

Since $\Psi\left(\tau, \tau^{2}, \lambda\right) \in \operatorname{co}\left\{\Psi(0,0, \lambda), \Psi(T, 0, \lambda), \Psi\left(T, T^{2}, \lambda\right)\right\}$, $\forall \tau \in[0, T],(32)$ holds if

$$
\begin{aligned}
\Psi(0,0, \lambda) & <0 \\
\Psi(T, 0, \lambda) & <0 \\
\Psi\left(T, T^{2}, \lambda\right) & <0 .
\end{aligned}
$$

Because (35) implies (34), the conditions (33)-(35) are sufficient to guarantee the stability with the proposed switching law.

\section{Appendix C: ProOf of COROllary 1}

Proof: Continuing with the proof of Theorem 2, the inequality (32) can be separated as two parts: one with the weighting factor $w_{1}$ and $w_{2}$ and the other without.

$$
\left[\begin{array}{cccc}
0 & 0 & 0 & 0 \\
* & 0 & 0 & 0 \\
* & * & 0 & 0 \\
* & * & * & -\rho T-\varepsilon \frac{w_{2}}{w_{1}}
\end{array}\right]+\Delta<0
$$

$\Delta$ represents the inequality without the weighting factor $w_{1}$ and $w_{2}$. For simplicity, the details of the $\Delta$ is omitted.

Obviously, the inequality (36) with a given $w_{1}>0$ and any $w_{2}>0$ holds, if the inequality (36) with a given $w_{1}>0$ and $w_{2}=0$ holds. Hence, when the inequalities (11) hold, Theorem 2 is satisfied.

\section{APPENDIX D: PROOF OF COROLLARY 2}

Proof: Continuing with the proof of Theorem $2, \Omega\left(\tau, \tau^{2}, \lambda\right)$ is the only term dependent on $\lambda$. By substituting $A(\lambda)=\lambda A_{1}+$ $(1-\lambda) A_{2}$ into $\Omega\left(\tau, \tau^{2}, \lambda\right)$ and rearranging the matrix, one can have:

$$
\Omega\left(\tau, \tau^{2}, \lambda\right)=\lambda M+(1-\lambda) N=\lambda(M-N)+N
$$

where

$$
\begin{aligned}
M & =\left[\begin{array}{cccc}
A_{1}^{T} P_{j}+P_{j} A_{1} & 0 & -\tau\left(A_{1}^{T} P_{j}+P_{j} A_{1}\right) & 0 \\
* & 0 & 0 & 0 \\
* & * & \tau^{2}\left(A_{1}^{T} P_{j}+P_{j} A_{1}\right) & 0 \\
* & * & * & 0
\end{array}\right] \\
N & =\left[\begin{array}{cccc}
A_{2}^{T} P_{j}+P_{j} A_{2} & 0 & -\tau\left(A_{2}^{T} P_{j}+P_{j} A_{2}\right) & 0 \\
* & 0 & 0 & 0 \\
* & * & \tau^{2}\left(A_{2}^{T} P_{j}+P_{j} A_{2}\right) & 0 \\
* & * & * & 0
\end{array}\right] .
\end{aligned}
$$


Because $\lambda \in\left[\lambda_{\min }, \lambda_{\max }\right]$, we can define:

$$
\lambda=p \lambda_{\text {max }}+(1-p) \lambda_{\min }, p \in[0,1] .
$$

The condition (2) can be written as:

$$
\begin{aligned}
\Lambda(0,0)+\beta\left(\lambda_{\min }(M-N)+N\right) & <0 \\
\Lambda\left(T, T^{2}\right)+\beta\left(\lambda_{\min }(M-N)+N\right) & <0 \\
\Lambda(0,0)+\beta\left(\lambda_{\max }(M-N)+N\right) & <0 \\
\Lambda\left(T, T^{2}\right)+\beta\left(\lambda_{\max }(M-N)+N\right) & <0,
\end{aligned}
$$

where $\Lambda\left(\tau, \tau^{2}\right)=\Pi(0)+\beta \Omega(0,0)-\sum_{r}^{\Xi} \mu_{j r} \Upsilon_{j}(0,0)$.

Then multiplying $(1-p)$ to (41)-(42) and multiplying $p$ to (43)-(44), one obtains:

$$
\begin{aligned}
(1-p) \Lambda(0,0)+\beta\left((1-p) \lambda_{\text {min }}(M-N)+p N\right) & <0 \\
(1-p) \Lambda\left(T, T^{2}\right)+\beta\left((1-p) \lambda_{\text {min }}(M-N)+p N\right) & <0 \\
p \Lambda(0,0)+\beta\left(p \lambda_{\text {max }}(M-N)+(1-p) N\right) & <0 \\
p \Lambda\left(T, T^{2}\right)+\beta\left(p \lambda_{\text {max }}(M-N)+(1-p) N\right) & <0 .
\end{aligned}
$$

Summing up (45)-(47) and (46)-(48) respectively yields,

$$
\begin{array}{r}
\Psi(0,0, \lambda)=\Lambda(0,0)+\beta(\lambda(M-N)+N)<0 \\
\Psi\left(T, T^{2}, \lambda\right)=\Lambda\left(T, T^{2}\right)+\beta(\lambda(M-N)+N)<0 .
\end{array}
$$

Now we can claim, if the conditions (2) hold, the conditions (10) hold for $\forall \lambda \in\left[\lambda_{\min }, \lambda_{\max }\right]$.

\section{ApPendix E: Proof of Theorem 3}

Proof: Similar to the proof of Theorem 1, the condition (16) yields:

$$
V_{1}(\phi(t), t) \leq e^{-2 \xi t} V_{1}(\phi(0), 0)+2 \beta \int_{0}^{t} e^{-2 \xi(t-s)} d s,
$$

which means that for $t \rightarrow \infty, x(t)$ exponentially converges to the region $\hat{\Theta}$.

We assume that at time $t, \sigma=i$ and $V_{1}(\phi(t))=$ $\min _{j \in \Xi}\left\{\phi(k)^{T} P_{j} \phi(t)\right\}=\phi(t)^{T} P_{j} \phi(t)$. Then along the solution of (1), the derivative of the chosen Lyapunov functional (6) can be calculated:

$$
\begin{aligned}
\dot{V}_{1} & =\min \left\{2 \phi ^ { T } ( t ) P _ { j } \left(\left(A_{i}+\sum_{m=1}^{q} \delta_{i}^{m} \hat{A}_{i}^{m}\right) \phi(t)\right.\right. \\
& \left.\left.+\left(A_{i}+\sum_{m=1}^{q} \delta_{i}^{m} \hat{A}_{i}^{m}\right) x_{e}+\left(B_{i}+\sum_{m=1}^{q} \delta_{i}^{m} \hat{B}_{i}^{m}\right)\right)\right\} \\
& =2 \phi^{T}(t) P_{j}\left(\left(A_{i}+\sum_{m=1}^{q} \delta_{i}^{m} \hat{A}_{i}^{m}\right) \phi(t)\right. \\
& \left.+\left(A_{i}+\sum_{m=1}^{q} \delta_{i}^{m} \hat{A}_{i}^{m}\right) x_{e}+\left(B_{i}+\sum_{m=1}^{q} \delta_{i}^{m} \hat{B}_{i}^{m}\right)\right)
\end{aligned}
$$

Define $\hat{\eta}(t)=\left[\begin{array}{lll}\phi(t) & x_{e} & 1\end{array}\right]^{T}$ and using convexity arguments, the condition (16) holds if

$$
\left[\begin{array}{cc}
\left(A_{i}+\hat{A}_{i}^{m}\right)^{T} P_{j}+P_{j}\left(A_{i}+\hat{A}_{i}^{m}\right)^{T}+\xi P_{j} & P_{j}\left(B_{i}+\hat{B}_{i}^{m}\right)+P_{j} \hat{A}_{i}^{m} x_{e} \\
* & -\beta
\end{array}\right]
$$

According to the switching law (14), similar to (27), the following inequality stands:

$$
2 \phi^{T}(k) P_{j}\left(A(\lambda)-A_{i}\right) \phi(k)-2 \phi^{T}(k) P_{j}\left(A_{i} x_{e}+B_{i}\right) \geq 0,
$$

Applying the S-procedure, the conditions (52) (53) hold if (15) holds.

\section{ACKNOWLEDGMENT}

We gratefully acknowledge the support of Dr. M. Yuratich and the team of TSL Technology Ltd. during this project.

\section{REFERENCES}

[1] D. Gomes, I. Barbi, and T. B. Lazzarin, "High voltage power supply using t-type parallel resonant dc-dc converter," IEEE Transactions on Industry Applications, 2018.

[2] R. Krishnan, Permanent magnet synchronous and brushless DC motor drives. CRC press, 2017.

[3] D. Lu and V. G. Agelidis, "Photovoltaic-battery-powered DC bus system for common portable electronic devices," IEEE Trans. Power Electron., vol. 24, no. 3, pp. 849-855, 2009.

[4] R. Orsagh, D. Brown, M. Roemer, T. Dabnev, and A. Hess, "Prognostic health management for avionics system power supplies," in Aerospace Conference, 2005 IEEE, pp. 3585-3591. IEEE, 2005.

[5] P. Midya, K. Haddad, and M. Miller, "Buck or boost tracking power converter," IEEE Power Electron. Lett., vol. 2, no. 4, pp. 131-134, 2004.

[6] O. García, M. Vasic, P. Alou, J. Oliver, J. Cobos et al., "An overview of fast DC-DC converters for envelope amplifier in RF transmitters," IEEE Trans. Power Electron., vol. 28, no. 10, pp. 4712-4722, 2013.

[7] R. Middlebrook and S. Cuk, "A general unified approach to modelling switching-converter power stages," in Power Electronics Specialists Conference, 1970 IEEE, pp. 18-34. IEEE, 1970.

[8] L. Corradini, D. Maksimović, P. Mattavelli, and R. Zane, Digital Control of High-Frequency Switched-Mode Power Converters. John Wiley \& Sons, 2015.

[9] J. Wang, S. Li, J. Yang, B. Wu, and Q. Li, "Extended state observerbased sliding mode control for pwm-based dc-dc buck power converter systems with mismatched disturbances," IET Control Theory \& Applications, vol. 9, no. 4, pp. 579-586, 2015.

[10] R. Ling, D. Maksimovic, and R. Leyva, "Second-order sliding-mode controlled synchronous buck dc-dc converter," IEEE Transactions on Power Electronics, vol. 31, no. 3, pp. 2539-2549, 2016.

[11] R. Anand, T. A. Samuel, and P. M. Mary, "Improved dynamic response of isolated full bridge $\mathrm{dc}$ to $\mathrm{dc}$ converter using bata optimization tuned fuzzy sliding mode controller for solar applications," International Journal of Hydrogen Energy, vol. 42, no. 34, pp. 21 648-21 658, 2017.

[12] S.-C. Tan, Y. Lai, C. K. Tse, and M. K. Cheung, "Adaptive feedforward and feedback control schemes for sliding mode controlled power converters," IEEE Trans. Power Electron., vol. 21, no. 1, pp. 182-192, 2006.

[13] C. Zhang, J. Wang, S. Li, B. Wu, and C. Qian, "Robust control for PWMbased DC-DC buck power converters with uncertainty via sampled-data output feedback," IEEE Trans. Power Electron., vol. 30, no. 1, pp. 504 $515,2015$.

[14] P. Karamanakos, T. Geyer, and S. Manias, "Direct voltage control of DC-DC boost converters using enumeration-based model predictive control," IEEE Trans. Power Electron., vol. 29, no. 2, pp. 968-978, 2014.

[15] S. Bibian and H. Jin, "High performance predictive dead-beat digital controller for DC power supplies," IEEE Trans. Power Electron., vol. 17, no. 3 , pp. 420-427, 2002.

[16] H. Maruta, M. Motomura, and F. Kurokawa, "A novel timing control method for neural network based digitally controlled DC-DC converter," in Power Electronics and Applications (EPE), 2013 15th European Conference on, pp. 1-8. IEEE, 2013.

[17] H. Li, F. Ren, J. Shang, B. Zhang, J. Lü, and H. Qi, "A novel large-signal stability analysis approach based on semi-tensor product of matrices with lyapunov stability theorem for dc-dc converters," in Energy Conversion Congress and Exposition (ECCE), 2016 IEEE, pp. 1-5. IEEE, 2016.

[18] R. Gavagsaz-Ghoachani, M. Phattanasak, J.-P. Martin, S. Pierfederici, B. Nahid-Mobarakeh, and P. Riedinger, "A lyapunov function for switching command of a dc-dc power converter with an lc input filter," IEEE Transactions on Industry Applications, vol. 53, no. 5, pp. 50415050, 2017. 
[19] M. Integrated, "Digital adjustment of DC-DC converter output voltage in portable applications," [Online] Available: http://www.maximintegrated.com/app-notes/index.mvp/id/818, 2002.

[20] Y. Wang, Q. Jin, and X. Ruan, "Optimized design of the multilevel converter in series-form switch-linear hybrid envelope-tracking power supply," IEEE Transactions on Industrial Electronics, vol. 63, no. 9, pp. 5451-5460, 2016

[21] M. Vasic, O. Garcia, J. Á. Oliver, P. Alou, D. Diaz, and J. A. Cobos, "Multilevel power supply for high-efficiency RF amplifiers," IEEE Trans. Power Electron., vol. 25, no. 4, pp. 1078-1089, 2010.

[22] D. Hammil, J. Deane, and D. Jeferries, "Modeling of chaotic DC-DC converters by iterative nonlinear mappings," IEEE Trans. Circuits and Systems Part I, vol. 35, no. 8, pp. 25-36, 1992.

[23] "Benefits and challenges of high-frequency regulators," Texas Instruments, Application Report, vol. AN-1973, 2013.

[24] B. Arbetter, R. Erickson, and D. Maksimovic, "Dc-dc converter design for battery-operated systems," in Power Electronics Specialists Confer ence, 1995. PESC'95 Record., 26th Annual IEEE, vol. 1, pp. 103-109. IEEE, 1995.

[25] R. Erickson and D. Maksimovic, "High efficiency DC-DC converters for battery- operated systems with energy management," in Worldwide Wireless Communications, Annual Reviews on Telecommunications, 1995.

[26] R. Priewasser, M. Agostinelli, C. Unterrieder, S. Marsili, and M. Huemer, "Modeling, control, and implementation of DC-DC converters for variable frequency operation," IEEE Trans. Power Electron., vol. 29 , no. 1, pp. 287-301, 2014

[27] A. Mallik and A. Khaligh, "Variable-switching-frequency state-feedback control of a phase-shifted full-bridge de/dc converter," IEEE Transactions on Power Electronics, vol. 32, no. 8, pp. 6523-6531, 2017.

[28] A. Filippov, Differential Equations with Discontinuous Righthand Sides. Springer, 1988

[29] B. J. Patella, A. Prodić, A. Zirger, and D. Maksimović, "High-frequency digital PWM controller IC for DC-DC converters," IEEE Trans. Power Electron., vol. 18, no. 1, pp. 438-446, 2003.

[30] D. Shevitz and B. Paden, "Lyapunov stability theory of nonsmooth systems," IEEE Transactions on automatic control, vol. 39, no. 9, pp. 1910-1914, 1994.

[31] G. S. Deaecto, J. C. Geromel, F. Garcia, and J. Pomilio, "Switche affine systems control design with application to DC-DC converters," IET control theory \& applications, vol. 4, no. 7, pp. 1201-1210, 2010

[32] X. Yan, Z. Shu, and S. M. Sharkh, "Prediction-based sampled-data control for dc-dc buck converters," in Smart Grid and Renewable Energy (SGRE), 2015 First Workshop on, pp. 1-6. IEEE, 2015.

[33] R. W. Erickson and D. Maksimovic, Fundamentals of Power Electronics. Springer Science \& Business Media, 2007.

[34] D. Mitchell and B. Mammano, "Designing stable control loops," in Proc. Unitrode Design Seminars, pp. 1-31, 2001

[35] K. Gu, "An integral inequality in the stability problem of time delay systems," in Proc of the 39th IEEE Conf on Decision and Control. Sydney, vol. 280522810, 2000.

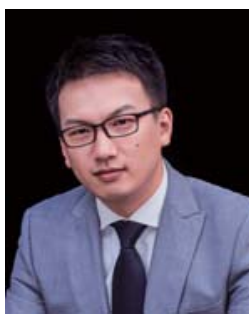

Xingda Yan (M'18) was born in 1989. He received the B.Eng. degree in automation from Harbin Institute of Technology, Harbin, China, in 2012, and the Ph.D. degree in electrical engineering from the University of Southampton, Southampton, U.K in 2017.

$\mathrm{He}$ is now a research fellow with the mechanical engineering department, University of Southampton, Southampton, U.K. His research interests include power electronics, hybrid system modelling and control, model predictive control, hybrid electric vehicle modelling and energy management.

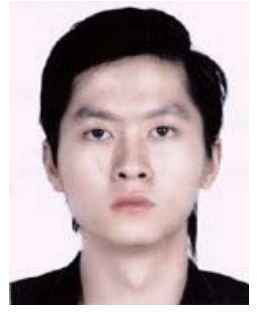

Zhan Shu (S'04-M'10-SM'18) was born in Nanchang, Jiangxi province, China, in 1982. He received his B.Eng. degree in Automation from Huazhong University of Science and Technology (HUST) in 2003, and the Ph.D. degree in Control Engineering from The University of Hong Kong (HKU) in 2008.

$\mathrm{He}$ was a postdoctoral researcher in the Hamilton Institute, National University of Ireland, Maynooth, and now is a lecturer in Faculty of Engineering and Physical Sciences, University of Southampton. He is a Senior Member of IEEE and an invited reviewer of Mathematical Review of the American Mathematical Society. He serves as an Associate Editor for Mathematical Problems in Engineering, an Associate Editor for Asian Journal of Control, an Associate Editor for Journal of The Franklin Institute, an Associate Editor of Proc. IMechE, Part I: J. Systems and Control Engineering, an Associate Editor of IET Electronics Letters, and a member of the IEEE Control Systems Society Conference Editorial Board. His current research interests include hybrid systems, positive systems, robust control, decentralized control, estimation and filtering, reinforcement learning, control application in power electronics, systems biology with emphasis on cell regulating networks and immunology.

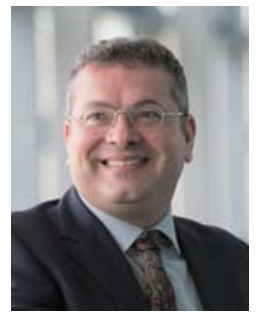

Suleiman M. Sharkh (SM'14) received the B.Eng. and Ph.D. degrees in electrical engineering from the University of Southampton, Southampton, U.K., in 1990 and 1994, respectively.

$\mathrm{He}$ is Professor of Power Electronics, Machines and Drives at the University of Southampton. $\mathrm{He}$ is also the Managing Director of $\mathrm{HiT}$ Systems Ltd. He has published over 160 papers in academic journals and conferences. His main research interests are in the area of control, electrical machine and power electronics with applications to electric vehicles, marine propulsion, exhaust energy recovery and submersible pumps. Prof. Sharkh is a senior member of the IEEE, a member of the IET and a Chartered Engineer. He was the 2008 winner of The Engineer Energy Innovation Award for his work on rim driven thrusters and marine turbine generators.

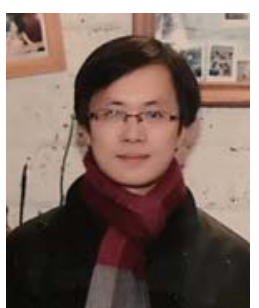

Zheng-Guang Wu (M'17) was born in 1982. He received the B.S. and M.S. degrees in Mathematics from Zhejiang Normal University, Jinhua, China, in 2004 and 2007, respectively, and the Ph.D. degree in control science and engineering from Zhejiang University, Hangzhou, China, in 2011.

$\mathrm{He}$ is currently with the Institute of Cyber Systems and Control, Zhejiang University. His current research interests include hybrid systems, networked systems and computational in-

telligence.

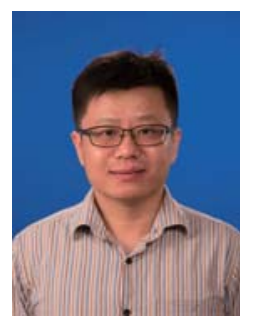

Michael Z. Q. Chen (M'08-SM'16) received the B.Eng. degree in electrical and electronic engineering from Nanyang Technological University, Singapore, and the Ph.D. degree in control engineering from Cambridge University, Cambridge, U.K.

$\mathrm{He}$ is currently a Professor at the School of Automation, Nanjing University of Science and Technology, Nanjing, China. He is a Guest Associate Editor for the International Journal of Bifurcation and Chaos. His current research interest is mechanical and electrical system control. 